\title{
Performance Analysis of Collaborative Hybrid-ARQ Protocols over Fading Channels
}

\author{
Igor Stanojev, Osvaldo Simeone and Yeheskel Bar-Ness \\ Center for Wireless Communications and Signal Processing Research \\ New Jersey Institute of Technology \\ Newark, New Jersey 07102-1982, USA \\ E-mail: \{iss2, osvaldo.simeone.2, yeheskel.barness\}@njit.edu
}

\begin{abstract}
Collaborative communications is a promising paradigm for future communications systems. In order to increase the spectral efficiency of collaborative schemes, an adaptive approach that prescribes the use of cooperation only upon erroneous reception of the transmitted packet by the destination has been recently proposed. This technique can be considered as a generalization of $\mathrm{ARQ}$ protocols to a collaborative scenario.

In this paper, a performance analysis is presented for both Collaborative Hybrid-ARQ Type I (i.e., without memory) and Chase Combining (i.e., with memory). In particular, a closed form expression for the average number of retransmissions and throughput is derived for single-relay case. Morever, numerical results are presented in order to corroborate the analysis and get insight into the performance of the considered schemes.
\end{abstract}

\section{INTRODUCTION}

Collaborative communications is envisioned to play an essential role as a new paradigm for future generation systems. This technology promises to improve the performance of current cellular, Wireless Local Area Networks (WLAN) or ad hoc/sensor networks by exploiting the diversity and power gains attainable with multiple radio terminals collaborating for transmission. This technology is particularly attractive in scenarios where deployment of multiple antennas on single terminals is not feasible. In case of perfect cooperation, the expected performance is that of a multiple antenna transmitter, which has been thoroughly studied in the last decade [1]. The more practical case where cooperation involves the allocation of radio resources (power, bandwidth) is currently under investigation. In particular, researchers have first focused on general models for terminals' collaboration (see, e.g., [2]) and then introduced realistic constraints, such as the inability of relay terminals to receive and transmit simultaneously (half duplex constraint [3]).

Most of the collaborative schemes proposed so far under the half duplex constraint exploit a fixed Time-Division-MultipleAccess (TDMA) frame structure for cooperation between terminals. For instance, for the single relay channel, a possible scheme allows the source terminal to transmit in the first time-slot whereas the source and relay terminal share the second time-slot (see, e.g., [4]). As recognized in [3], this approach may cause a reduction in the transmission rate, that could be overcome by using the help of a relay station only when needed. In other words, whenever the direct transmission between the source and the destination is not successful, the destination may require a retransmission and, in case a helping relay terminal is available, the latter can then cooperate with the source during retransmission. This approach is clearly a generalization of the Automatic-Repeat-reQuest (ARQ) protocol to a collaborative scenario and has been the subject of a few references [5].

In this paper, we tackle the analysis of Collaborative HybridARQ (HARQ) protocol described in [5], in terms of the expected number of transmissions needed for successful decoding and the average achievable throughput. Analysis is performed for single-relay Decode-and-Forward (DF) networks employing Hybrid ARQ Type I or Chase Combining (HARQT1 or HARQ-CC, respectively) protocols.

\section{SYSTEM OVERVIEW}

\section{A. Protocol Overview}

The transmission protocol is illustrated in fig. 1. During the first time-slot, the source broadcasts a packet that is decoded at the destination and the relay. If the destination erroneously decodes the packet (detected through the Cyclic Redundancy Check, CRC), it sends a Not-Acknowledge (NACK) feedback message to request retransmission. If at the same time the relay decodes the message successfully, as in example depicted by fig. 1, it signals its availability to the source and switches from the receiving to the transmitting mode. In this case, the source and the activated relay collaborate for the transmission by sending a Space-Time-Block-Code (STBC) codeword in the next transmission slot [4]. This joint retransmission is decoded by the destination following the STBC decoding rules. The procedure repeats until final successful transmission, confirmed by the destination via an Acknowledge (ACK) message.

Two types of HARQ protocols are considered, namely HARQ-TI and HARQ-CC. In the first, upon the reception of the NACK message, the source and, if activated, the relay retransmit a copy of the original Forward-Error-Correction (FEC) protected packet and the erroneous packets at the destination are discarded. On the other hand, according to the HARQ-CC protocol, previously received erroneous packets at the destination are preserved for combining with currently received packet. In particular, the summation is performed over the outputs of STBC decoder from all available (re)transmissions. 


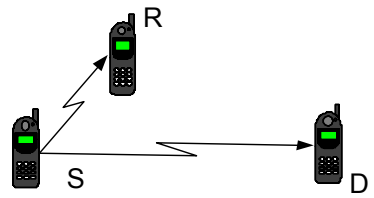

O riginal transm is sion

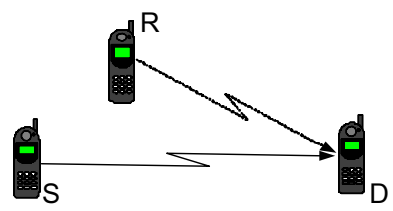

Retransm is sion
Fig. 1. Illustration of single-relay collaborative ARQ. In this example, relay $R$ decodes succesfully the original transmission and cooperates with the source $\mathrm{S}$ for retransmission.

\section{B. System Model}

We consider a system consisting of the source, the relay and the destination nodes equipped with a single antenna. A block Rayleigh fading model is assumed, where the channel stays constant during the transmission slot, but changes independently with each retransmission. The channel gains between any two nodes (the source, the relay and the destination) are modeled as mutually independent identically distributed symmetric complex Gaussian variables with the unit power. The transmission power $P$ is equal for both the source and the relay and the single-sided power spectral density of AdditiveWhite-Gaussian-Noise (AWGN) at any receiver is $N_{0}$. The model of interest in this paper places the source and the relay relatively close to each other, at approximately the same distance from the destination. Therefore, the channel power gain between source and relay is assumed to be $\alpha>1$ times larger than the channel power gains between source and destination and between relay and destination. Channel-StateInformation (CSI) is assumed at the receiver and not at the transmitter, unless specifically mentioned. Moreover, feedback channels from the destination toward the source and the relay are modelled as perfectly reliable for the transmission of short ACK/NACK messages. Their transmission time, as well as propagation and processing delays are considered negligible and are therefore not accounted for.

\section{Performance Analysis}

The average system delay can be parameterized with the expected number of transmissions, $E[T]$, where $T$ is the actual number of transmissions necessary for the successful decoding at the destination node

$$
E[T]=\sum_{n=1}^{\infty} n P\{T=n\}
$$

In (1), the probability that exactly $n$ transmissions are needed, $P\{T=n\}$, is given by

$$
P\{T=n\}=\left[1-p_{e}(n)\right] \prod_{k=1}^{n-1} p_{e}(k),
$$

with $p_{e}(k)$ denoting the probability that $k$ th transmission was erroneous, given that the previous transmissions were also unsuccessful. Erroneous transmission is defined as the event when the achievable rate $C$ is smaller than the transmission rate $C_{0}[\mathrm{nat} / \mathrm{s} / \mathrm{Hz}], C<C_{0}$. Note that, according to (1)-(2), in order to determine the delay and the average throughput, defined as the ratio $C_{0} / E[T]$, it is sufficient to solve for the probability of error $p_{e}(k)$. The following analysis of considered Collaborative HARQ protocols is therefore focused on derivation of $p_{e}(k)$.

\section{A. HARQ-TI}

The rate achievable at the relay after $k$ transmissions is

$$
C_{R}(k)=\log \left(1+\left|h_{S R}^{(k)}\right|^{2} \frac{\alpha P}{N_{0}}\right),
$$

with $h_{S R}^{(k)}$ denoting the channel gain between the source and the relay in the $k$ th transmission. Introducing the notation $\left\{C(1: k)<C_{0}\right\}$ for the event $\left\{C(1)<C_{0}, \ldots, C(k)<C_{0}\right\}$, we can express the probability that the relay has not yet correctly decoded at the $k$ th transmission as

$$
\begin{aligned}
\bar{p}_{R}(k) & =P\left\{C_{R}(1: k)<C_{0}\right\} \\
& =\prod_{i=1}^{k} P\left\{C_{R}(i)<C_{0}\right\} .
\end{aligned}
$$

Since in (3), the channel power gain $\left|h_{S R}^{(i)}\right|^{2}, i=1,2, . . k$, is a chi-square variable with two degrees of freedom, (4) becomes

$$
\bar{p}_{R}(k)=F_{\chi^{2}}^{k}(\mu / \alpha, 2),
$$

where $\mu=2 \frac{e^{C_{0}}-1}{P / N_{0}}, F_{\chi^{2}}(x, 0)=1$ and $F_{\chi^{2}}(x, \nu), \nu=$ $1,2, \ldots$ is the cumulative distribution function of the chi-square variable with $\nu$ degrees of freedom, taken at value $x$. On the other hand, the probability that the relay has decoded correctly at the $k$ th attempt is

$$
\begin{aligned}
p_{R}(k) & =P\left\{C_{R}(1: k-1)<C_{0}\right\}\left[1-P\left\{C_{R}(k)<C_{0}\right\}\right] \\
& =F_{\chi^{2}}^{k-1}(\mu / \alpha, 2)\left[1-F_{\chi^{2}}(\mu / \alpha, 2)\right] .
\end{aligned}
$$

Depending on whether the relay is activated or not, the achievable rate at the destination node at the $k$ this attempt is respectively

$$
\begin{aligned}
& C_{D}(k ; R)=\log \left[1+\left(\left|h_{S D}^{(k)}\right|^{2}+\left|h_{R D}^{(k)}\right|^{2}\right) \frac{P}{N_{0}}\right] \\
& C_{D}(k ; \bar{R})=\log \left[1+\left|h_{S D}^{(k)}\right|^{2} \frac{P}{N_{0}}\right]
\end{aligned}
$$

with $h_{S D}^{(k)}$ and $h_{R D}^{(k)}$ denoting the channel gains between the source and the destination, and between the relay and the destination in the $k$ th transmission, respectively. Note that diversity of degree two in (7a) originates from STBC transmission from two antennas (the source and the relay), and results in an equivalent channel power gain $\left|h_{S D}^{(k)}\right|^{2}+\left|h_{R D}^{(k)}\right|^{2}$ that is a chi-square variable with four degrees of freedom. The probability of error at the $n$th attempt can be written now as

$$
\begin{aligned}
p_{e}(n)= & \sum_{k=1}^{n-1} p_{R}(k) P\left\{C_{D}(n ; R)<C_{0}\right\} \\
& +\bar{p}_{R}(n-1) P\left\{C_{D}(n ; \bar{R})<C_{0}\right\} .
\end{aligned}
$$


Combining (5) and (6) with (8), we finally have

$$
\begin{aligned}
p_{e}(n)= & \left(1-F_{\chi^{2}}^{n-1}(\mu / \alpha, 2)\right) F_{\chi^{2}}(\mu, 4) \\
& +F_{\chi^{2}}^{n-1}(\mu / \alpha, 2) F_{\chi^{2}}(\mu, 2) .
\end{aligned}
$$

\section{B. HARQ-CC}

At the $k$ th transmission, the rate achievable by the relay is

$$
C_{R}(k)=\log \left(1+\sum_{i=1}^{k}\left|h_{S R}^{(i)}\right|^{2} \frac{\alpha P}{N_{0}}\right),
$$

where the equivalent channel power gain $\sum_{i=1}^{k}\left|h_{S R}^{(i)}\right|^{2}$ is a chi-square variable with $2 k$ degrees of freedom. Moreover, the probability that after $k$ transmissions the relay still did not receive the message successfully can be written as

$$
\begin{aligned}
\bar{p}_{R}(k) & =P\left\{C_{R}(1: k)<C_{0}\right\} \\
& =P\left\{C_{R}(k)<C_{0}\right\},
\end{aligned}
$$

or, according to (10),

$$
\bar{p}_{R}(k)=F_{\chi^{2}}(\mu / \alpha, 2 k),
$$

and the probability that the relay received it successfully at the trial $k$, but not before, reads

$$
\begin{aligned}
p_{R}(k)= & P\left\{C_{R}(1: k-1)<C_{0}\right\} \times \\
& {\left[1-P\left\{C_{R}(k)<C_{0} \mid C_{R}(1: k-1)<C_{0}\right\}\right] } \\
= & P\left\{C_{R}(k-1)<C_{0}\right\} \times \\
& {\left[1-P\left\{C_{R}(k)<C_{0} \mid C_{R}(k-1)<C_{0}\right\}\right] } \\
= & F_{\chi^{2}}(\mu / \alpha, 2(k-1))\left[1-\frac{F_{\chi^{2}}(\mu / \alpha, 2 k)}{F_{\chi^{2}}(\mu / \alpha, 2(k-1))}\right] \\
= & F_{\chi^{2}}(\mu / \alpha, 2(k-1))-F_{\chi^{2}}(\mu / \alpha, 2 k) .
\end{aligned}
$$

Unlike HARQ-TI, for the HARQ-CC protocol the destination rate depends not only on whether the relay is transmitting, but also on the instant when it was activated. Therefore, instead of using $C_{D}(k ; \bar{R})$ and $C_{D}(k ; R)$, we switch to the notation $C_{D}(k ; j)$, where $j$ represents the transmission slot when the relay successfully decodes the message

$$
C_{D}(k ; j)=\log \left[1+\left(\sum_{i=1}^{k}\left|h_{S D}^{(i)}\right|^{2}+\sum_{i=j+1}^{k}\left|h_{R D}^{(i)}\right|^{2}\right) \frac{P}{N_{0}}\right] .
$$

Note that the the equivalent channel power gain $\sum_{i=1}^{k}\left|h_{S D}^{(i)}\right|^{2}+\sum_{i=j+1}^{k}\left|h_{R D}^{(i)}\right|^{2}$ defined in (14), valid only for $j \leq k-1$, is a chi-square variable with $(2 k-j)$ degrees of freedom. For the case when the relay was not able to receive before the current retransmission, we use the notation $C_{D}(k ; k)$ :

$$
C_{D}(k ; k)=\log \left(1+\sum_{i=1}^{k}\left|h_{S D}^{(i)}\right|^{2} \frac{P}{N_{0}}\right) .
$$

Finally, the probability of error at the $n$th transmission is

$$
\begin{gathered}
p_{e}(n)=\sum_{k=1}^{n-1} p_{R}(k) P\left\{C_{D}(n ; k)<C_{0} \mid C_{D}(1: n-1 ; k)<C_{0}\right\} \\
+\bar{p}_{R}(n-1) P\left\{C_{D}(n ; n)<C_{0} \mid C_{D}(1: n-1 ; n-1)<C_{0}\right\} \\
\quad=\sum_{k=1}^{n-1} p_{R}(k) P\left\{C_{D}(n ; k)<C_{0} \mid C_{D}(n-1 ; k)<C_{0}\right\} \\
+\bar{p}_{R}(n-1) P\left\{C_{D}(n ; n)<C_{0} \mid C_{D}(n-1 ; n-1)<C_{0}\right\} .
\end{gathered}
$$

Combining the last equation with (12) and (13) gives us the final result for the probability of error conditioned on previous unsuccessful attempts:

$$
\begin{aligned}
p_{e}(n)= & \sum_{k=1}^{n-1}\left\{\left[F_{\chi^{2}}(\mu / \alpha, 2(k-1))-F_{\chi^{2}}(\mu / \alpha, 2 k)\right] \times\right. \\
& \left.\frac{F_{\chi^{2}}(\mu, 2(2 n-k))}{F_{\chi^{2}}(\mu, 2(2 n-k-2))}\right\}+ \\
& F_{\chi^{2}}(\mu / \alpha, 2(n-1)) \frac{F_{\chi^{2}}(\mu, 2 n)}{F_{\chi^{2}}(\mu, 2(n-1))} .
\end{aligned}
$$

\section{Numerical Results}

The influence of parameter $\alpha$ on the system performance is illustrated in fig. 2, that shows the average throughput, i.e., $C_{0} / E[T]$, versus average signal to noise ratio, $S N R=P / N_{0}$, for Collaborative HARQ-CC scheme. Moreover, upper and lower bounds on the performance of a single-relay collaborative systems, namely the average throughputs of a $2 \times 1 \mathrm{MISO}$ (Multiple-Input-Single-Output, i.e., ideal collaboration) and a $1 \times 1$ SISO (Single-Input-Single-Output, i.e., no collaboration) networks using HARQ-CC, respectively, are also shown. It is clear that increasing the gain $\alpha$ improves the performance in the low $S N R$ regime, where the throughput of Collaborative HARQ is comparable to the performance of the ideal $2 \times 1$ system. In fact, for low $S N R$, the number of retransmissions is large and therefore the advantage of the $2 \times 1$ system of achieving double spatial diversity degree in the very first transmission attempt is negligible. On the other hand, for large $S N R$ the number of retransmissions is small and Collaborative HARQ approaches the performance of a $1 \times 1$ system.

Fig. 3 compares the average throughput of collaborative to that of $2 \times 1$ and $1 \times 1$ network using HARQ-TI and HARQCC protocols versus the $S N R$, for $\alpha=20 d B$. For each of the three network types, HARQ-CC clearly outperforms HARQ-TI in the low $S N R$ region, since the large number of retransmissions allows systems with memory to show their full potential. However, as the $S N R$ increases, the advantage of HARQ-CC over HARQ-TI protocol decreases.

The influence of transmission rate $C_{0}$ on the average throughput for Collaborative HARQ-CC scheme is presented in fig. 4. Large transmission rates fully exploit the system resources in the large $S N R$ regime, but can lead to the system overload in the low $S N R$ region. On the other hand, small transmission rates show excellent performance in the low $S N R$ regions, but are unable to exploit the benefits of high 


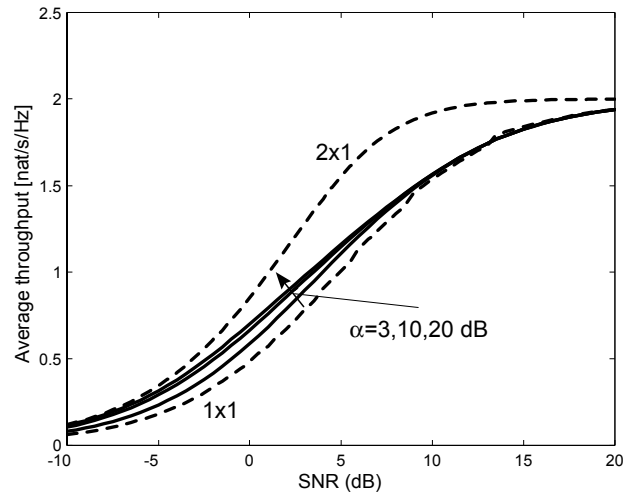

Fig. 2. Average throughput versus $S N R$ for different $\alpha$ (HARQ-CC scheme, $C_{0}=2 \mathrm{nat} / \mathrm{sec} / \mathrm{Hz}$ )

$S N R$. The envelope of the fixed-rate curves (solid line), which shows the maximum throughputs at each $S N R$ value, depicts the possible performance of the system when it adaptively selects the transmission rate $C_{0}$, based on the knowledge of the average $S N R$ at the transmitter.

Fig. 5 extends the example depicted in fig. 3 by showing the average throughputs for the collaborative, the $2 \times 1$ and the $1 \times 1$ systems for adaptive rate scheme mentioned above. The ability to adapt the transmission rate yields a relatively low expected number of retransmissions. Therefore, as shown in this figure, following the previous discussion, collaborative HARQ performs close to the $1 \times 1$ lower bound, while HARQCC provides a throughput similar to HARQ-TI.

\section{CONCLUSION}

In this paper a mathematical framework has been provided for the analysis of the single-relay Collaborative HARQ protocols. Extensive numerical results based on the analysis have been presented in order to provide the insight into the performance of considered systems. In future work, analysis of HARQ-IR (Incremental Redundancy) protocols, as well as of multi-relay systems will be investigated.

\section{ACKNOWLEDGMENT}

This work was supported in part by Samsung Advanced Institute of Technology (SAIT), Korea.

\section{REFERENCES}

[1] A. Goldsmith, S. A. Jafar, N. Jindal and S. Vishwanath, "Capacity Limits of MIMO Channels," IEEE Journ. Selected Areas Commun., vol. 21, no. 5, pp. 684-702, June 2003.

[2] A. Sendonaris, E. Erkip and B. Aazhang, "User cooperation diversityPart I: System description," IEEE Trans. Commun., vol. 51, pp. 19271938, Nov. 2003.

[3] J. N. Laneman, D. N. C. Tse and G. W. Wornell, "Cooperative Diversity in Wireless Networks: Efficient Protocols and Outage Behavior," IEEE Trans. Inform. Theory, vol. 50, no. 12, pp. 3062-3080, Dec. 2004.

[4] R. U. Nabar, H. Bölcskei and F. W. Kneubühler, "Fading relay channels: performance limits and space-time signal design," IEEE Journ. Selected Areas Commun., vol. 22, no. 6, pp. 1099-1109, Aug. 2004.

[5] B. Zhao and M. C. Valenti, "Practical Relay Networks: A Generalization of Hybrid-ARQ," IEEE Journ. Selected Areas Commun., vol. 23, no. 1, pp. 7-18, Jan. 2005.

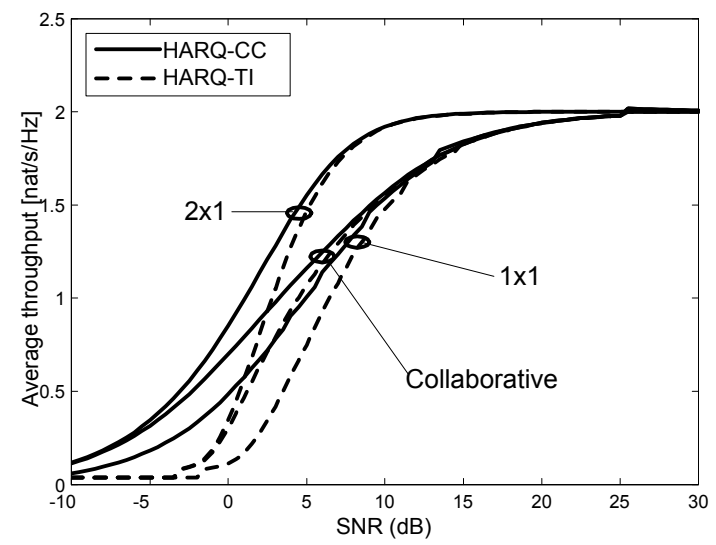

Fig. 3. Average throughput versus $S N R$ for different network types and HARQ protocols $\left(\alpha=20 d B, C_{0}=2 \mathrm{nat} / \mathrm{s} / \mathrm{Hz}\right)$.

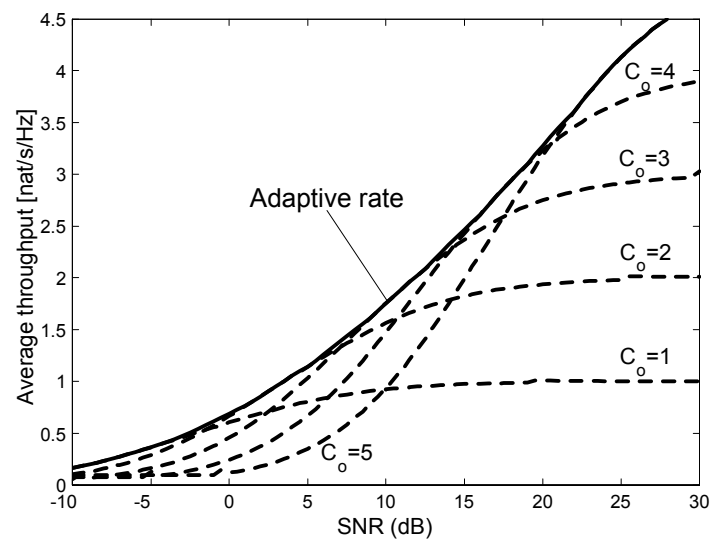

Fig. 4. Average throughput of systems with fixed transmission rates and of the system with the adaptive rate allocation ability (Collaborative HARQ-CC scheme, $a=20 d B$ ).

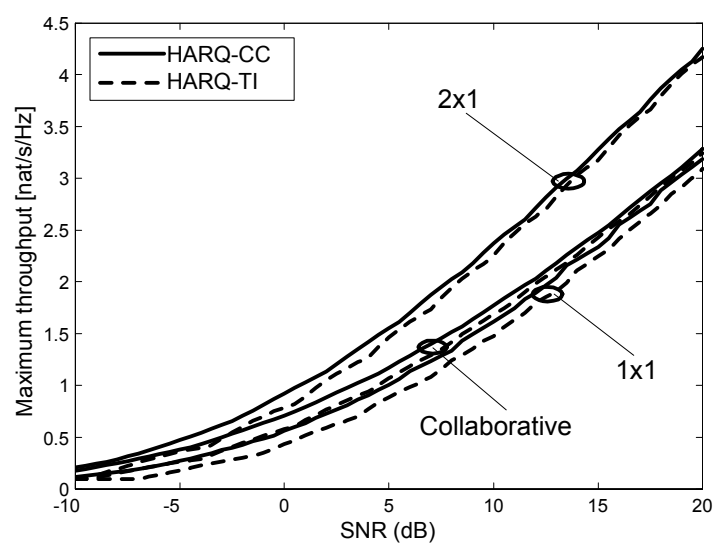

Fig. 5. Average throughput with adaptive rate allocation based on the average $S N R$ at the transmitter $(\alpha=20 d B)$. 